\title{
Recommendations on Adapting Crowdsourcing to Problem Types
}

\author{
Mahmood Hosseini, Alimohammad Shahri, Keith Phalp, Raian Ali \\ Faculty of Science and Technology \\ Bournemouth University, UK \\ \{mhosseini, ashahri, kphalp, rali\}@bournemouth.ac.uk
}

\begin{abstract}
Crowdsourcing is a paradigm which enables and harnesses the power and wisdom of a usually large, diverse crowd in innovation, problem solving and knowledge acquisition. The scale, benefits and application areas of this traditional model are amplified by the advances of information and communication technology such as the advent of Web 2.0, which, at the same time, has increased the complexity and, hence, the need for systematic development approaches. While crowdsourcing has been successfully applied in several projects and de-facto platforms already exist for them, the research on engineering principles, methods and tools for developing a crowdsourcing project is still in the early stages. In this paper, we study the adaptation of crowdsourcing settings to fit the nature of the problem being crowdsourced. As a method, we review the literature and complement that with an online expert survey involving practitioners and researchers active in the field of crowdsourcing. We then interpret the obtained results and identify a set of recommendations on how to set up crowdsourcing to fit each of the five common categories of problems. Our results inform future crowdsourcing developers with best practice experiences on planning and configuring their projects.
\end{abstract}

Keywords-Engineering Crowdsourcing, Adaptive Crowdsourcing, Crowdsourcing Configuration

\section{INTRODUCTION}

Crowdsourcing is a form of innovation seeking, problem solving and knowledge acquisition paradigm which is typically based on the power of voluntary participation of a usually large, diverse number of people for performing tasks within loose contractual settings through an open call. In that sense, crowdsourcing can be viewed as a weaker form of outsourcing and it usually implies a minimised degree of control over the entire process, including the recruitment of the crowd and their performance and job quality. It also implies a high level of uncertainty which makes its unsuitable or risky for certain problems and for certain preferences and constraints of crowdsourcers.

While crowdsourcing is not a new concept, its feasibility and popularity have been amplified through advances in information and communication technology. Crowdsourcing was coined as a term and revisited as a concept in light of the current technology in the seminal work of Howe in [1]. The popularity of social media and the increased familiarity of the public with information sharing and online collaborative work are other facilitators of crowdsourcing. Consequently, several crowdsourcing commercial platforms started to appear, such as Amazon Mechanical Turk [2], and Threadless [3], and the number of participants is increasing for these new ways of performing lightweight jobs.
A great deal of emphasis has been given to the application of crowdsourcing for problem solving in different domains. The main argument is to prove its feasibility and fitness to the peculiarities of these domain. For example, [4] discusses the utilisation of the crowdsourcing concept in the domain of environmental sciences and shows its ability to solve problems related to water resources management. For another example, [5] demonstrates how crowdsourcing could be exploited for problems related to planetary surface mapping. Furthermore, some studies on crowdsourcing argue that crowdsourcing is not an alternative to automation but the only solution if the problem is too complex to be solved by computers, such as cognitive problems [6].

To maximise the quality of crowdsourcing activities, engineering principles and approaches are needed. The literature has studied various aspects, including ethical issues [7], managing collaboration and coordination [8], and pricing [9] in crowdsourcing activities. Amongst the wide range of open questions that exist on the utilisation of crowdsourcing, configuration of a crowdsourcing project to fit the nature of the crowdsourced problem needs to be explored. For example, for a certain crowdsourced task we may need to decide whether to recruit a competent crowd or a crowd with different levels of skills.

In this paper, we develop a set of best practice recommendations on setting up a crowdsourcing project to fit the nature of the crowdsourced problems. We study five crowdsourcing problem types that are typically found in the crowdsourcing literature. To derive those recommendations, we draw on the literature together with a survey involving practitioners and researchers in the domain of crowdsourcing. Besides the main results related to crowdsourcing customisation to the problem types, we elaborate on a set of debates for which there has been no clear answer in previous research or current practices. Therefore, this could become a starting point for future research in the area.

The rest of the paper is organised as follows. In Section III we present an overview of the problem under study. In Section III. we describe the methodology which we followed for conducting our study. In Section IV] we present the results of our study and reflect on them. In Section $\mathrm{V}$, we discuss our findings from the expert survey and also discuss the threats to the validity of our study. We conclude our paper in Section VI and suggest future work. 


\section{BACKGROUND}

Crowdsourcing is an old model of obtaining knowledge and solving problems by the crowd. As a term, however, it was coined in 2006 [1]. The popularity of crowdsourcing has recently increased, both because of the widespread and ease of use of current information and communication technology and because of the ever-increasing familiarity of the public with different methods of sharing knowledge, e.g., through social media. However, there is still a lack of engineering methods as how to arrange, design and set up a crowdsourcing project [10]. Consequently, this has inspired various studies targeting a set of facets with regard to crowdsourcing, for example studies in the design and management of crowdsourcing platforms [11], [12], studies in the quality and validity of the obtained data [13], [14], and studies in the legal and ethical perspectives of crowdsourcing [7], [15], [16].

The lack of engineering methods is, to some extent, because of the lack of theoretical backgrounds on the notion of crowdsourcing since its appearance in the literature. However, a few studies have been conducted recently to address this issue. For instance, [17] propose a taxonomic theory of crowdsourcing, [18] suggests a preliminary taxonomy of the nature of crowdsourcing, [19] provide a taxonomy of crowdsourcing processes, and [20] discuss the conceptual foundations of crowdsourcing.

We already proposed a taxonomy for crowdsourcing in [21]. This taxonomy was extracted and informed by analysing 113 research papers in which the concept of crowdsourcing was clearly defined. The proposed taxonomy helped define the four pillars of crowdsourcing which are present in any typical crowdsourcing project:

- The crowd: the people who participate in a crowdsourcing activity by bringing their expertise, knowledge, innovation, money, etc. to that activity

- The crowdsourcer: the people, organisations, or companies with problems to outsource to the crowd

- The crowdsourced task: the problem introduced by the crowdsourcer which needs a solution by the crowd

- The crowdsourcing platform: the online service that facilitates the performance of the crowdsourced task

These four pillars have a subset of features or, in the case of crowdsourcing platform, a subset of facilities, hereafter also referred to as features. Based on the crowdsourcing activity and the context, some of these features can be optional, i.e., the selection of these features depends on the needs of the crowdsourcer and the characteristics of the crowdsourced task. These features were also extracted from definitions in the 113 papers. A full description of this taxonomy can be found on [21].

Given the large subset of features for crowdsourcing, it proposes the problem of configuration in crowdsourcing platforms. Wrong configuration settings can adversely affect the crowd in terms of the number of participants, their required diversity, their motivation to participate, and their privacy inclinations. It can also adversely affect the crowdsourcers in terms of their resources, and their desired quantity and quality of the obtained results. Ultimately, it can adversely affect the crowdsourcing platform itself in terms of difficulties in the management and supervision of the crowd and crowdsourcers and exposure to misuse.

To solve the configuration problem in crowdsourcing platforms, it is necessary to develop a crowdsourcing product configurator tool. Such a tool can automatically provide configuration information tailored to the needs of every crowdsourcing activity. However, and as explained before, theoretical research is needed prior to implementation in order to remove misconduct and partial solutions to the problem. This paper intends to provide such theoretical foundation, by referring to the literature and experts' opinion and building upon what we already proposed in [21].

\section{Methodology}

The purpose of this study is to find best practice guidelines on how to configure crowdsourcing for popular problem types. In this section, we describe these problem types and how we conducted the expert opinion studies to obtain those best practice recommendations.

\section{A. Classifying the Problem Types}

While surveying the literature, we could identify five common types of problems in the current practice of crowdsourcing. In this study, we focus on these problem types as explained in the following:

1) Recruiting participants for opinion collection tasks, e.g., an online survey. The participants should answer different questions by choosing the answer they think is correct. The main characteristics of this activity is as follows:

- The problem type is opinion acquisition

- There is no right or wrong answer

2) Recruiting participants for a basic task, e.g., a simple online object recognition where the participants are asked to count the number of stones they see in different pictures. The main characteristics of this activity is as follows:

- The problem type is easy and simple

- No special knowledge or expertise is required

3) Recruiting participants for tasks that require expertise, e.g., an online astronomy project where the participants are expected to have a working knowledge of celestial bodies. The main characteristics of this activity is as follows:

- The problem type is difficult and complex

- Expertise in the subject is required before contributing anything meaningful

4) Recruiting participants for competitive tasks where the reward is given to some participants and not all, e.g., an online transcription project where the participants are asked to transcribe some videos, and only one transcription (presumably the most correct one) will be incentivised. The main characteristics of this activity is as follows:

- The problem type can be easy or difficult

- There is a chance that the participant will get no tangible incentives 
TABLE I. CROWDSOURCING PROBLEM TYPES AND THEIR CHARACTERISTICS

\begin{tabular}{ccccc}
\hline $\begin{array}{c}\text { Problem } \\
\text { Type }\end{array}$ & $\begin{array}{c}\text { Correct } \\
\text { Answers? }\end{array}$ & $\begin{array}{c}\text { Task } \\
\text { Simplicity }\end{array}$ & $\begin{array}{c}\text { Expertise } \\
\text { Required? }\end{array}$ & Incentives \\
\hline $\begin{array}{c}\text { Opinion-Based } \\
\text { Problems }\end{array}$ & No & Varied & Yes/No & (un)limited \\
\hline $\begin{array}{c}\text { Basic } \\
\text { Problems }\end{array}$ & Yes & Simple & No & (un)limited \\
\hline $\begin{array}{c}\text { Complex } \\
\text { Problems }\end{array}$ & Yes & Difficult & Yes & (un)limited \\
\hline $\begin{array}{c}\text { Competition } \\
\text { Type Problems }\end{array}$ & Yes & Varied & Yes/No & limited \\
\hline $\begin{array}{c}\text { Collaborative } \\
\text { Fundraising }\end{array}$ & No & Simple & No & (un)limited \\
\hline
\end{tabular}

5) Recruiting participants for collaborative donation tasks, e.g., a crowdfunding project where the participants are requested to donate a non-compulsory amount of money (with a minimum set) to a charity programme. The main characteristics of this activity is as follows:

- Everyone can contribute

- It needs willingness and motivation for participants to donate their money

The five crowdsourcing problem types and some of their characteristics are summarised in Table 1 .

\section{B. Experts Survey}

To conduct this study, we prepared an online expert survey using the five crowdsourcing problem types that we described earlier together with the taxonomy that we proposed in [21]. Each problem type was described at the beginning, and then the list of crowdsourcing features was presented to the experts. The experts were asked to choose those crowdsourcing features that they deemed useful for the given crowdsourcing problem type. The list of crowdsourcing features, which was the topic of discussion in our online expert system, is given in Table II To view a complete list of crowdsourcing features, how they were extracted, and how a taxonomy was built around them, please refer to our previous work [21].

TABLE II. CROWD, CROWDSOURCER, AND CROWDSOURCING PLATFORM FEATURES

\begin{tabular}{cccc}
\hline $\begin{array}{c}\text { Crowd } \\
\text { Features }\end{array}$ & $\begin{array}{c}\text { Crowdsourcer } \\
\text { Features }\end{array}$ & \multicolumn{2}{c}{$\begin{array}{c}\text { Crowdsourcing } \\
\text { Platform }\end{array}$} \\
\hline \multirow{2}{*}{ Diversity } & Financial & Provide & Provide \\
& Incentives & Enrolment & Feedback Loops \\
\hline \multirow{2}{*}{ Unknown-ness } & Social & Provide & Provide Quality \\
& Incentives & Authentication & Threshold \\
\hline \multirow{2}{*}{ Largeness } & Entertainment & Provide Skill & $\begin{array}{c}\text { Provide Quantity } \\
\text { Threshold }\end{array}$ \\
& Incentives & Declaration & The \\
& Open & Provide Task & Manage \\
\multirow{2}{*}{ Undefined-ness } & Call & Assignment & Platform Misuse \\
\hline \multirow{2}{*}{ Competence } & Privacy & Supervise & Provide \\
& Provision & Crowd & Ease of Use \\
\hline \multirow{2}{*}{ Motivation } & Feedback & Provide Task & $\begin{array}{c}\text { Provide } \\
\end{array}$ \\
& to Crowd & Broadcast & Attraction \\
\cline { 2 - 4 } & & Provide Price & Provide Payment \\
& & Negotiation & Mechanism \\
\hline
\end{tabular}

To identify the experts for our survey, we used Google Scholar to identify those crowdsourcing experts with at least three related quality publications. The expert survey was invitation-only to ensure the quality of the results. A total of 139 experts were invited by email to participate in our survey. They had different backgrounds and domain expertise to ensure diversity. 50 experts replied to our invitation, while only 37 of them actually completed the survey. In this paper, we only report the responses and opinions of the experts who completed the survey. The experts' affiliations type and major expertise in crowdsourcing are presented in Table III.

TABLE III. SUMMARY OF EXPERTS' INFORMATION

\begin{tabular}{lcc}
\hline $\begin{array}{l}\text { Type of Expertise } \\
\text { in Crowdsourcing }\end{array}$ & $\begin{array}{c}\text { Major Expertise } \\
\text { in Crowdsourcing }\end{array}$ \\
\hline Academia & 32 & $\begin{array}{c}\text { Social sensing, Crowdfunding, Citizen Science Project, } \\
\text { Crowdsourcing for multimedia quality assessment, } \\
\text { Trust and privacy in crowd-enhanced systems, } \\
\text { Structured volunteer crowdsourcing, }\end{array}$ \\
Industry & 1 & Quality assurance in crowdsourcing, Micro tasking platforms \\
Both & 4 & .
\end{tabular}

\section{RESUlTS}

In this section, we report and reflect on experts' opinions gathered from the online expert survey on the importance of the selection of crowdsourcing features for different crowdsourcing problem types. We report the results based on the problem types we presented in section III-A with the crowdsourcing features presented Table III In this survey, crowdsourcing features are reported within the following classification based on the number of the experts' votes:

- Trivial Features: We define trivial features as those features with votes fewer than 20 per cent (votes of seven experts or fewer), and when there was also no argument given by any of the experts or the literature on the essential nature of the feature for the problem type.

- Insignificant Features: We define insignificant features as those features with votes between 20 per cent and 50 per cent (votes of eight experts to 18 experts), and when there was also no argument given by any of the experts or the literature on the essential nature of the feature for the problem type.

- Important Features: We define important features as those features with votes between 50 per cent and 80 per cent (votes of 19 experts to 29 experts), and when there was an argument why the feature should be considered important for the problem type given by experts or the literature.

- Critical Features: We define critical features as those features with votes more than 80 per cent (votes of 30 experts or more), and when there was an argument why the feature should be considered critical for the problem type is given by experts or the literature.

Although we put these criteria, it is worth mentioning that the percentages were good representatives of the importance of a feature and were consistent with the experts' arguments and our analysis of the literature.

\section{A. Problem Type 1: Crowdsourcing for Opinion Collection Tasks}

In this problem type, the experts were asked about the degree of importance of crowdsourcing features for the tasks in which the crowd who participate are asked for their opinions, e.g., an online survey. This problem type requires participants to voice their opinions and there are no right or wrong answers. This means that all the collected data are valid. 
1) Crowd features: The experts stated that largeness and diversity are the critical features in this crowdsourcing problem type. This is backed up by the fact that opinion collection tasks usually target a wide audience, and their results are only generalisable when diversity, along with largeness of the sample, is ensured. Motivation was considered an important feature, as participants without motivation may easily opt out of the activity. Randomness in the crowd was the least selected feature, illustrating that opinion collection tasks usually target a specific audience, e.g., people belonging to certain communities, certain age groups, certain competencies in a field, etc.

2) Crowdsourcer features: It was stated by the experts that providing financial incentives is a critical feature for this category of problems. Opinion collection tasks usually have a low response rate [22], and financial incentives, e.g., vouchers and prize draws, can enhance the response rate [23]. All the other crowdsourcer features were considered to be important, with feedback being voted for the highest in this group. This may illustrate that some participants may actually be interested in the results of an opinion collection task, and by providing them the results, they are incentivised and more motivated for future participation.

3) Crowdsourcing platform features: Experts selected platform ease of use as a crucial feature. Payment mechanisms, attractive platform, platform misuse management, quality and quantity threshold, feedback loops, authentication and task broadcast were the important features. This shows the importance of platform design for opinion collection tasks, as it can attract more participants and result in a higher response rate. All other platform features were deemed insignificant.

The results of the expert survey illustrating the importance of crowdsourcing features in opinion collection tasks are shown in table IV

TABLE IV. IMPORTANCE OF CROWDSOURCING FEATURES IN OPINION-BASED CROWDSOURCING PROBLEMS

\begin{tabular}{|c|c|}
\hline \multicolumn{2}{|r|}{ Opinion-Based Problems } \\
\hline $\begin{array}{l}\text { Critical } \\
\text { Features }\end{array}$ & $\begin{array}{l}\text { Ease of Use (32), Largeness (31), Diversity (30), } \\
\text { Financial Incentives (30) }\end{array}$ \\
\hline $\begin{array}{l}\text { Important } \\
\text { Features }\end{array}$ & $\begin{array}{l}\text { Payment Mechanism (28), Feedback Provision (25), Attraction (25), } \\
\text { Open Call (24), Quality Threshold (24), } \\
\text { Platform Misuse Management (24), Social Incentives (23), } \\
\text { Feedback Loops (23), Entertainment Incentives (21), } \\
\text { Authentication (21), Quantity Threshold (21), Motivation (20), } \\
\text { Task Broadcast (19), Privacy Provision (19) }\end{array}$ \\
\hline $\begin{array}{c}\text { Insignificant } \\
\text { Features }\end{array}$ & $\begin{array}{l}\text { Enrolment (18), Skill Declaration (17), Task Assignment (17), } \\
\text { Unknown-ness (16), Competence (16), Supervise Crowd (15), } \\
\text { Undefined-ness (11), Price Negotiation (9) }\end{array}$ \\
\hline $\begin{array}{l}\text { Trivial } \\
\text { Features }\end{array}$ & None \\
\hline
\end{tabular}

\section{B. Problem Type 2: Crowdsourcing for Basic Tasks}

In this problem type, the experts were asked about the degree of importance of crowdsourcing features for the tasks which require common sense and basic everyday knowledge, e.g., identifying the number of seashells in a photo which is an activity that does not require any particular experience or expertise from the participants.
1) Crowd features: Largeness was viewed as a critical feature of the crowd. This was to ensure that statistical methods reveal the correct answer, e.g., using mode of the obtained data to find the number of seashells in a photo, or using mean of the obtained data to find the weight of an object. Motivation was viewed as an important factor, while diversity was considered insignificant. This is a valid observation, as diversity plays a trivial role in tasks based on facts and not opinions.

2) Crowdsourcer features: Providing financial incentives was indicated to be a critical feature for such tasks. For example, participants in an online survey might be genuinely interested in the results of the survey, motivating them to take part even if no other incentives are provided. However, since simple tasks may have no other tangible benefits for the participants, financial incentives remain the only driving force for participants' recruitment. Providing entertainment incentives, providing feedback and recruitment through an open call were also considered to be important features.

3) Crowdsourcing platform features: No critical features were identified to be implemented in the platform relating to this crowdsourcing problem type. They, however, indicated the following features to be important in the design of the platform: providing attraction and ease of use, payment mechanisms, platform misuse management, quality and quantity threshold and feedback loops. Ease of use, quality threshold and payment mechanisms were especially noticed by the experts. This could be because simple tasks are more probable to be done by the common crowd, who would be confused and probably demotivated if the platform is not easy to use. Quality threshold was also selected because in this problem type, there is a correct answer.

The results of the expert survey illustrating the importance of crowdsourcing features in basic crowdsourcing tasks are shown in table $\mathrm{V}$

TABLE V. IMPORTANCE OF CROWDSOURCING FEATURES IN BASIC CROWDSOURCING PROBLEMS

\begin{tabular}{cl}
\hline & \multicolumn{1}{c}{ Basic Problems } \\
\hline $\begin{array}{c}\text { Critical } \\
\text { Features }\end{array}$ & Financial Incentives (33), Largeness (31) \\
\hline \multirow{3}{\text{Important}}{$\begin{array}{l}\text { Features } \\
\text { Entertainment Incentives (25), Platform Misuse Management (25), } \\
\text { Motivation (24), Feedback Provision (23), Feedback Loops (23), } \\
\text { Attraction (23), Open Call (21), Quantity Threshold (20) }\end{array}$} \\
\hline $\begin{array}{l}\text { Social Incentives (18), Diversity (17), Task Broadcast (17), } \\
\text { Ansignificant } \\
\text { Features }\end{array}$ & $\begin{array}{l}\text { Enrolment (13), Supervise Crowd (13), Task Assignment (12), } \\
\text { Undefined-ness (10), Privacy Provision (10), Price Negotiation (9), } \\
\text { Skill Declaration (8) }\end{array}$ \\
\hline Trivial & None \\
\hline
\end{tabular}

\section{Problem Type 3: Crowdsourcing for Tasks That Require Expertise}

In this problem type, the experts were asked about the degree of importance of crowdsourcing features for the tasks which require expertise or competence in a specific area of knowledge, e.g., an astronomy task where the crowd bring their astronomy expertise and experience. In this crowdsourcing problem type, there must be preconditions for selecting the right crowd in order to obtain trustworthy, quality results. 
1) Crowd features: It was stated that competence and motivation are critical features for the participating crowd. Expertise is needed in this problem type because without it, the obtained results will not have the desired quality. Motivated participants are essential because of the difficult nature of the task, i.e., unmotivated participants may lose their interest and focus in such challenging tasks and opt out of the activity. Similarly, anonymity and randomness were identified as trivial features that should probably be avoided. These features may adversely affect the quality of such challenging jobs and therefore should be contemplated on thoroughly before being selected.

2) Crowdsourcer features: It was indicated that giving feedback to the crowd and providing social incentives are critical features for the crowdsourcer. Feedback was deemed critical because participants in such activities are less inclined toward the incentives and more inclined toward the result of their participation. This also explains why financial incentives were not classified as critical. Providing privacy was also an insignificant feature, meaning that the crowdsourcer should have participants' information in order to be able to determine whether they are qualified for the task.

3) Crowdsourcing platform features: Experts listed the following features as important features for the platform: enrolment and authentication, skill declaration and task broadcast, feedback loops, quality threshold, platform misuse management, ease of use and attraction. Price negotiation mechanisms were least voted for, because as explained earlier, participants in such crowdsourcing activities are less inclined to participate for the money.

The results of the expert survey illustrating the importance of crowdsourcing features in complex, expertise-required crowdsourcing tasks are shown in table $\mathrm{VI}$.

TABLE VI. IMPORTANCE OF CROWDSOURCING FEATURES IN PROBLEMS THAT REQUIRE EXPERTISE

\begin{tabular}{|c|c|}
\hline \multicolumn{2}{|r|}{ Problems That Require Expertise } \\
\hline $\begin{array}{l}\text { Critical } \\
\text { Features }\end{array}$ & $\begin{array}{l}\text { Competence (33), Feedback Provision (32), Social Incentives (31), } \\
\text { Motivation (30) }\end{array}$ \\
\hline $\begin{array}{l}\text { Important } \\
\text { Features }\end{array}$ & $\begin{array}{l}\text { Quality Threshold (28), Skill Declaration (26), Feedback Loops (26), } \\
\text { Largeness (25), Ease of Use (25), Entertainment Incentives (24), } \\
\text { Attraction (24), Open Call (22), Enrolment (22), Authentication (21), } \\
\text { Platform Misuse Management (21), Financial Incentives (20), } \\
\text { Task Broadcast (19) }\end{array}$ \\
\hline $\begin{array}{l}\text { Insignificant } \\
\text { Features }\end{array}$ & $\begin{array}{l}\text { Task Assignment (18), Quantity Threshold (18), } \\
\text { Payment Mechanism (18), Supervise Crowd (17), Diversity (16), } \\
\text { Privacy Provision (10), Price Negotiation (10) }\end{array}$ \\
\hline $\begin{array}{c}\text { Trivial } \\
\text { Features }\end{array}$ & Unknown-ness (7), Undefined-ness (1) \\
\hline
\end{tabular}

\section{Problem Type 4: Crowdsourcing for Competitive Tasks}

In this problem type, the experts were asked about the degree of importance of crowdsourcing features for the tasks in which the crowd compete to get rewarded, e.g., a transcription project in which only one participant gets the incentives based on the quality of the provided solution. The task itself can range in difficulty and length.

1) Crowd features: Competence, motivation and largeness were selected to be three important features in this crowdsourcing problem type. It could be said that competence helps increase participants' motivation as it will help them in a finding more quality solution and therefore increases their chances of getting the incentive. Largeness actually helps the crowdsourcer in the selection of a better solution delivered by the crowd. Other features were chosen by the experts as insignificant features.

2) Crowdsourcer features: It was stated that providing financial incentives is a critical feature for such tasks, as participants may have no other motivation to take part in them. Providing social incentives, open call and feedback were among important features. Entertainment incentives and privacy provision were selected as insignificant features, indicating that such tasks may not attract participants if they are only amusing and without any tangible incentives. As for privacy, experts believed that crowdsourcers may need reputation systems to ensure the quality of the obtained result, therefore reducing the level of privacy and anonymity of participants.

3) Crowdsourcing platform features: Only four features were identified as insignificant: task assignment and broadcast, supervision of the crowd and price negotiation. All the other features were voted as important features. Two platform features, ease of use and payment mechanism were the most voted for, indicating their importance.

The results of the expert survey illustrating the importance of crowdsourcing features in competitive crowdsourcing tasks are shown in table VII

TABLE VII. IMPORTANCE OF CROWDSOURCING FEATURES IN COMPETITIVE PROBLEMS

\begin{tabular}{|c|c|}
\hline \multicolumn{2}{|r|}{ Competitive Problems } \\
\hline $\begin{array}{l}\text { Critical } \\
\text { Features }\end{array}$ & Financial Incentives (32) \\
\hline $\begin{array}{c}\text { Important } \\
\text { Features }\end{array}$ & $\begin{array}{l}\text { Payment Mechanism (28), Ease of Use (28), Competence (26), } \\
\text { Quality Threshold (26), Motivation (25), Feedback Provision (25), } \\
\text { Enrolment (22), Skill Declaration (22), Quantity Threshold (22), } \\
\text { Attraction (21), Social Incentives (21), Open Call (21), } \\
\text { Platform Misuse Management (20), Authentication (19), } \\
\text { Largeness (19), Feedback Loops (19) }\end{array}$ \\
\hline $\begin{array}{c}\text { Insignificant } \\
\text { Features }\end{array}$ & $\begin{array}{l}\text { Supervise Crowd (17), Task Assignment (16), } \\
\text { Entertainment Incentives (14), Diversity (13), } \\
\text { Task Broadcast (13), Price Negotiation (12), Unknown-ness (11), } \\
\text { Privacy Provision (9), Undefined-ness (8) }\end{array}$ \\
\hline $\begin{array}{l}\text { Trivial } \\
\text { Features }\end{array}$ & None \\
\hline
\end{tabular}

\section{E. Problem Type 5: Crowdsourcing for Collaborative Fundraising Tasks}

In this problem type, the experts were asked about the degree of importance of crowdsourcing features for the tasks in which the crowd should collaborate and donate, e.g., crowdfunding activities where participants bring their money. This type of task is usually simple, and no expertise is required for participation.

1) Crowd features: Largeness was seen as the only critical feature for the crowd. It is obvious that collaborative activities produce better results with a larger crowd. Diversity, anonymity and motivation were among important features. Competence was considered to be a trivial feature, since it adds no value to the crowdsourcing activity. 
2) Crowdsourcer features: Social incentives and open call were perceived as critical features of crowdsourcers. In such donation tasks, open calls are needed to attract as many participants as possible and social incentives should be provided to motivate those participants. Feedback was an important feature as participants in such activities are more motivated and satisfied when they know how their donations are spent and what results are achieved. The rest of the features were insignificant.

3) Crowdsourcing platform features: Experts identified the following features as trivial features: skill declaration, task assignment, supervision of crowd, price negotiation, quality threshold and payment mechanism. Ease of use and attraction were among important features, showing the importance of these features in attracting a larger crowd. Other important features were task broadcast, feedback loops and management of platform misuse. No platform features were considered to be critical.

The results of the expert survey illustrating the importance of crowdsourcing features in collaborative fundraising tasks are shown in table VIII. TABLE VIII. IMPORTANCE OF CROWDSOURCING FEATURES IN
COLLABORATIVE FUNDRAISING PROBLEMS

\begin{tabular}{cl}
\hline & \multicolumn{1}{c}{ Collaborative Fundraising Problems } \\
\hline $\begin{array}{c}\text { Critical } \\
\text { Features }\end{array}$ & Social Incentives (37), Largeness (33), Open Call (30) \\
\hline \multirow{2}{\text{Important}}{$\begin{array}{l}\text { Features } \\
\text { Motivation (27), Diversity (23), Feedback Loops (21), } \\
\text { Platform Misuse Management (20), Unknown-ness (20), } \\
\text { Task Broadcast (19) }\end{array}$} \\
\hline $\begin{array}{c}\text { Ensignificant } \\
\text { Features }\end{array}$ & $\begin{array}{l}\text { Entertainment (18), Undefined-ness (16), Privacy Provision (16), } \\
\text { Quantity Threshold (14), Financial Incentives (9) }\end{array}$ \\
\hline Trivial & $\begin{array}{l}\text { Price Negotiation (7), Payment Mechanism (7), } \\
\text { Quality Threshold (6), Supervise Crowd (5), }\end{array}$ \\
Features & Competence (3), Skill Declaration (3), Task Assignment (3) \\
\hline
\end{tabular}

\section{F. A Guide to Feature Selection Based on Crowdsourcing Problem Types}

The obtained information from the expert survey led to a crowdsourcing configuration setting, summarised in Table IX The table lists critical features in italics and important features in normal text. Each feature is followed by a number in brackets showing the number of experts' votes for that problem type. This can be used by both crowdsourcers and crowdsourcing platform developers as a guide to better facilitate their crowdsourcing activity.

\section{DISCUSSION}

In this section, we discuss the importance of crowdsourcing features in our study in relation to the crowdsourcing problem types. This section is meant to further develop our understanding of these features and their importance. We also discuss threats to the validity of our study.

\section{A. Crowd Features}

Diversity is usually considered to be a core feature of crowdsourcing [24]. However, in our study we found out that diversity is not always an essential feature, especially when the solutions to the crowdsourced task can be verified as right or wrong answers. On the other hand, when crowdsourcing is performed for obtaining opinions, innovations, collaboration, and funds, diversity was highly advocated.

Anonymity or unknown-ness was generally voted as an insignificant feature. This could relate to many problems that may arise when the crowd is unknown, such as information integrity problems [25] and fraudulent activities [26]. Anonymity was specifically discouraged in those crowdsourcing activities which need expertise. In these activities, skill declaration was also highly voted for, meaning that when the crowd provides their skills in a crowdsourcing platform, they are identifying and anonymising themselves to a certain extent.

Largeness is also usually considered to be a core feature of crowdsourcing [24]. Our study showed that it is always a critical or important feature. However, for competitive crowdsourcing activities, it was voted for less than other activities. This could arise from the competitive nature of the task. It also informs that engaging a large crowd in such activities will be a challenge for the crowdsourcers.

Randomness or undefined-ness was another crowd feature which was deemed either trivial or insignificant. It was generally believed that participation in crowdsourcing activities should be systematic, which could decrease the chances of obtaining low-quality information or inefficient solutions.

Competence of the crowd was voted high in activities which really need expertise. Simple online tasks and collaborative donation tasks had therefore a low voting for competence. This shows the importance of a crowdsourcing configurator which can engage the right crowd in the crowdsourcing activity.

Motivation as a crowd feature was always an important or critical feature. Such importance has led to several studies [27]-[29]. Therefore, motivation should be considered as a core element of crowdsourcing and as an essential feature of the participating crowd.

\section{B. Crowdsourcer Features}

Providing incentives for the crowd is usually considered to be an integral part of any crowdsourcing activity [30]. Financial incentives seem to be the dominant type of incentives, giving the driving force to many online crowdsourcing platforms such as Amazon Mechanical Turk. However, for tasks that need expertise and for tasks that need collaboration, social incentives outvoted financial incentives. On the other hand, financial incentives were deemed critical in simple crowdsourcing activities, while social incentives were seen as insignificant in such activities. Entertainment incentives were also voted as important in both simple and difficult tasks, however, they were voted insignificant in competitive and collaborative donation tasks.

Providing an open call for participants was also important in all crowdsourcing problem types. This means that crowdsourcing activities should be open to the general public. However, given the problem type, crowdsourcers may choose certain individuals in the crowd to fit their task and obtain better quality results. 
TABLE IX. FEATURE CLASSIFICATION ACCORDING TO THEIR DEGREE OF IMPORTANCE FOR PROBLEM TYPES

\begin{tabular}{|c|c|}
\hline Problem Type & Feature Classification \\
\hline $\begin{array}{l}\text { Opinion } \\
\text { Based } \\
\text { Problems }\end{array}$ & $\begin{array}{l}\text { Ease of Use (32), Largeness (31), Diversity (30), Financial Incentives (30) } \\
\text { Payment Mechanism (28), Feedback Provision (25), Attraction (25), } \\
\text { Open Call (24), Quality Threshold (24), Platform Misuse Management (24), } \\
\text { Social Incentives (23), Feedback Loops (23), Entertainment Incentives (21), } \\
\text { Authentication (21), Quantity Threshold (21), Motivation (20), } \\
\text { Task Broadcast (19), Privacy Provision (19) }\end{array}$ \\
\hline Basic Problems & $\begin{array}{l}\text { Financial Incentives (33), Largeness (31) } \\
\text { Ease of Use (29), Payment Mechanism (28), Quality Threshold (28), } \\
\text { Entertainment Incentives (25), Platform Misuse Management (25), } \\
\text { Motivation (24), Feedback Provision (23), Feedback Loops (23), } \\
\text { Attraction (23), Open Call (21), Quantity Threshold (20) }\end{array}$ \\
\hline $\begin{array}{l}\text { Problems That } \\
\text { Require } \\
\text { Expertise }\end{array}$ & $\begin{array}{l}\text { Competence (33), Feedback Provision (32), Social Incentives (31), } \\
\text { Motivation (30) } \\
\text { Quality Threshold (28), Skill Declaration (26), Feedback Loops (26), } \\
\text { Largeness (25), Ease of Use (25), Entertainment Incentives (24), } \\
\text { Attraction (24), Open Call (22), Enrolment (22), Authentication (21), } \\
\text { Platform Misuse Management (21), Financial Incentives (20), } \\
\text { Task Broadcast (19) }\end{array}$ \\
\hline $\begin{array}{l}\text { Competitive } \\
\text { Problems }\end{array}$ & $\begin{array}{l}\text { Financial Incentives (32) } \\
\text { Payment Mechanism (28), Ease of Use (28), Competence (26), } \\
\text { Quality Threshold (26), Motivation (25), Feedback Provision (25), } \\
\text { Enrolment (22), Skill Declaration (22), Quantity Threshold (22), } \\
\text { Attraction (21), Social Incentives (21), Open Call (21), } \\
\text { Platform Misuse Management (20), Authentication (19), } \\
\text { Largeness (19), Feedback Loops (19) }\end{array}$ \\
\hline $\begin{array}{l}\text { Collaborative } \\
\text { Fundraising } \\
\text { Problems }\end{array}$ & $\begin{array}{l}\text { Social Incentives (37), Largeness (33), Open Call (30) } \\
\text { Ease of Use (29), Attraction (28), Feedback Provision (28), } \\
\text { Motivation (27), Diversity (23), Feedback Loops (21), } \\
\text { Platform Misuse Management (20), Unknown-ness (20), } \\
\text { Task Broadcast (19) }\end{array}$ \\
\hline
\end{tabular}

Providing privacy was generally seen by the experts to be insignificant. By privacy, we mean giving the crowd the opportunity to remain anonymous. As discussed in Section 5.1, finding several problems with anonymous online working was the driving force for such a decision.

Providing feedback to the crowd was a crowdsourcer feature that was always voted as important. This means that crowdsourcers should provide the participants with the results of the crowdsourcing activity as a motivation factor. This also calls for feedback channels to be embedded in the crowdsourcing platform to automate such feedback provision.

\section{Crowdsourcing Platform Features}

Providing enrolment and authentication for crowd members showed a correlation in this study, being insignificant features in simple activities and donation activities. This means that such platforms may not need any form of identification from crowd members as long as the job is performed and their goals are realised.

Similarly, skill declaration and task assignment illustrated similar correlation, having the lowest votes in simple activities and donation activities. It can be argued that in absence of enrolment and authentication facilities in a crowdsourcing platform, skill declaration and task assignment is also impossible.

Supervising the crowd and price negotiation were two platform features that obtained constantly low votes. It can be concluded that by providing a good entry criteria for participants, the need for supervision during the crowdsourcing activity is minimised. Furthermore, giving the crowd a negotiation chair was denied and seemed insignificant by the experts, meaning that only the crowdsourcer has such a privilege.

Task broadcasting was considered to be an important feature for opinion acquisition, difficult tasks and donation activities. This is justified by the fact that diversity plays an important role in these crowdsourcing problem types. These results are in harmony with those about diversity as a feature of the crowd. In the same fashion, feedback loops were considered to be important in all problem types, also in harmony with providing feedback as a crowdsourcer feature.

Quality and quantity thresholds are two more platform features which were generally considered to be an important feature, except for donation activities where there is no quality to be measured and there is no limit to the quantity, meaning that the more participants, the more profitable the results. 
Platform misuse management was also considered to be an important platform feature in all these crowdsourcing activities, leading to the conclusion that it is a necessary facility that must be always considered to ensure the integrity of the obtained results, among other crowdsourcers' concerns.

Platform ease of use and attraction were always important or critical features as well. Given that the crowdsourcing platform is used by a diversity of individuals with different levels of expertise in online activities, these features remain indisputable features for involvement and retention of more participants.

Payment mechanism in a platform was only deemed trivial in donation activities, in line with providing financial incentives as a crowdsourcer feature. This means that crowdsourcing platforms should provide a strong payment mechanism to help crowdsourcers with incentivising the participating crowd.

\section{The Importance of Crowdsourcing Features in General}

It is also interesting to observe how crowdsourcing features were perceived generally, regardless of the crowdsourcing problem type. In this section, we will observe the results of the expert survey regarding this issue and reflect on these results and provide further insight into crowdsourcing features.

1) The Overall Importance of Crowd Features: Figure 1 shows the information we obtained from the experts about the importance of crowd features in general in all crowdsourcing problem types in all crowdsourcing problem types.

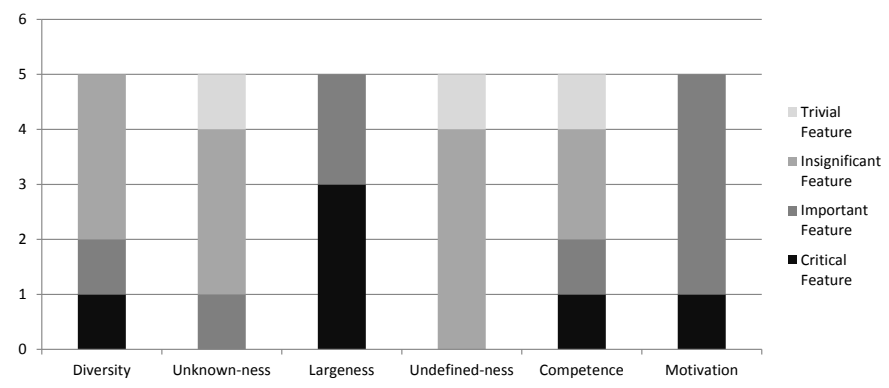

Fig. 1. Crowd features and their degree of importance regardless of crowdsourcing problem types

Based on the information provided by the experts in the expert survey, diversity was only deemed critical in opinionbased problem types, and important in collaborative fundraising problems. Diversity is indeed critical in opinion-based problem types as it allows a variety of viewpoints to be stated. In other problem types, it was an insignificant feature, which implies that it could be probably neglected by crowdsourcers. One reason could be that introducing diversity in other problem types could lead to difficulties in coordination.

Unknown-ness was only an important feature in collaborative fundraising problems, indicating that crowdsourcers must be willing to accept anonymous contributors, as their identity is not as much needed as their monetary contribution. In other problem types, it was either an insignificant feature or a trivial feature, indicating that crowdsourcers may choose to allow or not allow anonymous participants in their crowdsourcing activities.
Largeness was always seen either as a critical feature, or as an important feature. This is in line with the notion of crowdsourcing itself, which seeks to obtain the wisdom, expertise, and resources of as many people as possible. It also illustrates why the advent of web 2.0 has boosted the idea of crowdsourcing, as it allows people around the world to participate in crowdsourcing activities.

Undefined-ness was, on the other hand, always observed as an insignificant or trivial feature. This means that random selection of participants for crowdsourcing activities are either not important, for example in the case of collaborative fundraising problems or basic problems, or not advised, for example in the case of problems that require expertise.

Competence was also deemed critical in problems that require expertise, and important in competitive problems. This means that unless the crowdsourcing problem type does need expertise, it should not be a selection criterion for participants in crowdsourcing activities. Furthermore, in other problem types, it could limit the number of participants, and consequently, the number or amount of contribution.

Motivation was always a critical or important feature, indicating that crowdsourcers must always have methods of motivating participants in crowdsourcing activities regardless of the problem type. This could result in a larger crowd, more contributions, and more quality output.

2) The Overall Importance of Crowdsourcer Features: Figure 2 shows the information we obtained from the experts about the importance of crowdsourcer features in general.

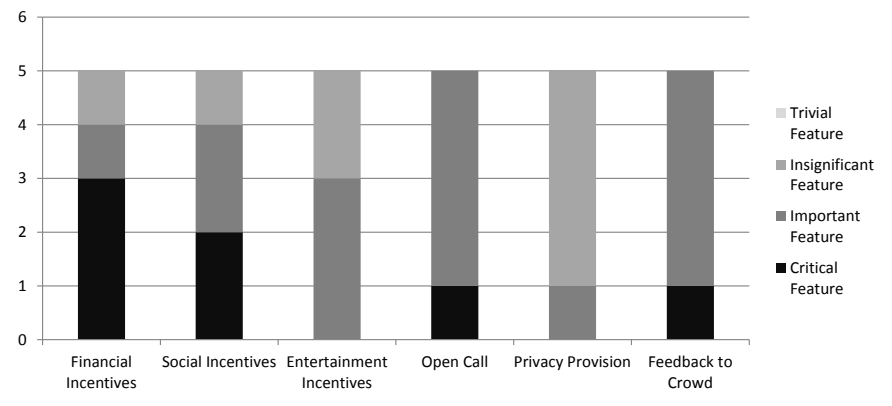

Fig. 2. Crowdsourcer features and their degree of importance regardless of crowdsourcing problem types

Based on the information provided by the experts in the expert survey, financial incentives were generally considered to be critical or important, except in the case of collaborative fundraising problems, as obviously it is the participants who should donate money and not the crowdsourcers. In this last case, social incentives were considered to be critical, which was also observed by the experts in our study. In all other problem types, however, it seems that financial incentives play an important role as a motivating element for the crowd to participate in crowdsourcing activities.

Social incentives were also generally considered to be critical or important, except in the case of basic crowdsourcing problems. This indicates that in basic problems, where almost everyone can be involved and solving the basic, easy problem does not provide any glory for the participant, financial 
incentives or entertainment incentives work much better than social incentives. In all other problem types, however, social incentives must be considered by crowdsourcers to attract more participants and probably get more quality contributions.

Entertainment incentives were considered to be important, except in competitive problems and collaborative fundraising problems. This indicates that when the crowdsourcing problem requires competition among participants or when the crowdsourcing problem is about collecting money from them, other forms of incentives, such as social incentives or financial incentives should be used and entertainment incentives work less efficiently and attract fewer participants. In other problem types, introducing entertainment incentives, such as gamification, can attract more participants.

Providing and open call was generally considered to be critical or important, indicating that crowdsourcers should allow all participants to take part in the crowdsourcing activity. However, it seems logical that crowdsourcers should later screen out those participants which do not match their selection criteria. In the case of collaborative fundraising problems, however, open call was considered to be critical as it is meant to attract as many participants as possible.

Privacy provision was generally considered to be an insignificant feature, except in the case of opinion-based problems. This was expected as this type of crowdsourcing activity reveals participants' opinions which may sometimes be of personal nature, while in all other crowdsourcing problem types the participants' contribution is based on facts or is their financial support.

Providing feedback to the crowd was always considered to be a critical or important feature, illustrating that crowdsourcers must always have methods in place to inform participants about their participation results, as this can affect their motivation for further participation.

3) The Overall Importance of Crowdsourcing Platform Features: Figures 3 and 4 shows the information we obtained from the experts about the importance of crowdsourcing platform features in general in all crowdsourcing problem types.

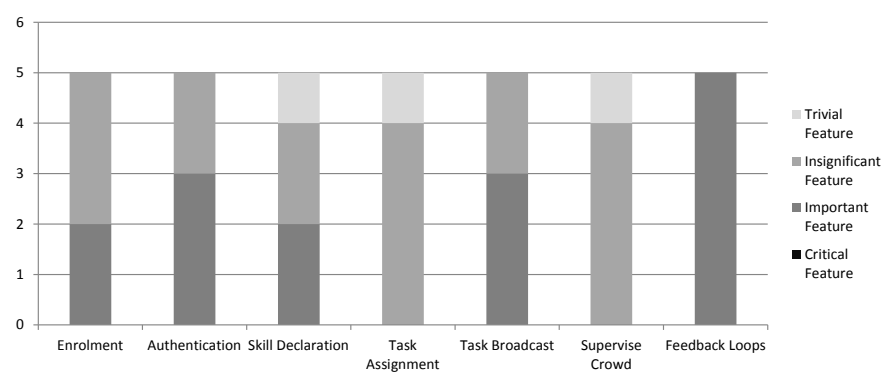

Fig. 3. Crowdsourcing platform features and their degree of importance regardless of crowdsourcing problem types, part 1

Based on the information provided by the experts in the expert survey, providing enrolment was considered to be important in competitive problem types and collaborative fundraising problem types, and in other problem types it was considered to be insignificant. In competitive problem types, enrolment can be important as you may need to record certain

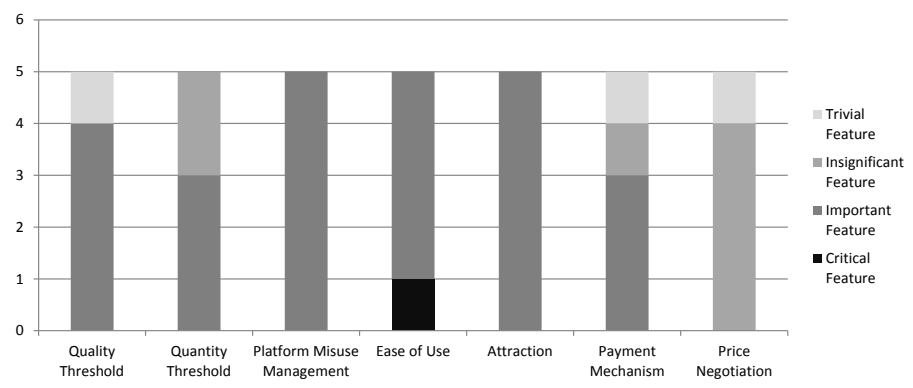

Fig. 4. Crowdsourcing platform features and their degree of importance regardless of crowdsourcing problem types, part 2

information about participants in the platform for future use, for example to reward them later. In collaborative fundraising problem types, enrolment could be used to track the amount of financial contribution.

Providing authentication was considered to be important in opinion-based problems, competitive problems and problems that require expertise, and it was considered to be insignificant in other problem types. In all these problem types, authentication could discourage and prevent malicious participants from joining in, and affecting the results of the crowdsourcing activity. In basic problems and collaborative fundraising collaborative problems, it was considered to be insignificant, because the first problem type deals with easyto-solve problems and malicious participants could easily be detected, and the second problem type would not attract malicious participants since participation in them requires donating money.

Providing skill declaration was considered to be an important feature in competitive problems and problems that require expertise, and it was insignificant or trivial in other problem types. These two problem types would benefit from skill declaration by participants as crowdsourcers could screen and select the them based on this information. Such declaration would be of little use in basic problems, where the problem would be solvable by almost any participants regardless of their skills, in opinion-based problems where crowdsourcers seek to obtain participants' opinions and not their skills, and in collaborative fundraising problems, where no skills are actually required.

Providing task assignment was generally considered to be either insignificant or trivial. This could show that crowdsourcing tasks can be assigned to every participant and then later results can be screened to choose only those which satisfy the requirements of the crowdsourcers.

Providing task broadcast was considered to be important in opinion-based problems, problems that require expertise and collaborative fundraising problems, and it was considered to be insignificant in other problem types. This could mean that basic problems and competitive problems do not benefit from a task broadcast mechanism in the platform, as the first one deals with easy-to-solve problems that generally is attractive to all due to its simplicity, and the second one works in a different way than broadcasting the task since the participants may need to be selected through a procedure.

Supervising the crowd was considered to be either insignif- 
icant or trivial, indicating that crowdsourcing platforms do not need to supervise participants during their participation, and should only screen them before they start to participate, and screen the results after their participation.

Providing feedback loop was always considered to be an important feature. Similar to feedback provision as a crowdsourcer feature, this clearly illustrates the need for the platform to enable crowdsourcers in their feedback provision to the crowd.

Providing quality threshold was a platform feature that was considered to be an important one, except in the case of collaborative fundraising tasks, where it was considered to be trivial. The latter is the case since collecting money from participants needs no quality controls. In all other problem types, there must be mechanisms in the platform to ensure the quality of participants' contribution.

Providing quantity threshold was a platform feature that was considered to be important in opinion-based problems, basic problems and problems that require expertise. In other task type it was considered to be insignificant. It is obviously insignificant in collaborative fundraising problems as the crowdsourcers prefer as many participants as they can get, so there must be no limit to the quantity. In problems that require expertise, it is also insignificant as even a small number of experts can produce the desired results that crowdsourcers are looking for. So there is no need to provide any limits to the quantity. In all other problem types, however, there must be a quantity threshold to prevent scattered, probably inconsistent results.

Managing platform misuse was another platform feature that was always considered to be important. This indicates that crowdsourcing platform developers must be aware of the possibility of malicious participants who may damage and sabotage the platform or the results, and provide mechanisms to prevent from such platform misuse.

Platform ease of use was generally considered to be an important or critical feature of the crowdsourcing platform. As the platform deals with the crowd, it is important for the platform to be designed in a way that can be used by everyone, so that all potential participants are empowered to participate in the crowdsourcing activity.

Platform attraction was also generally considered to be an important factor, indicating that the platform must be able to attract as many participants as possible by its design and usability features.

Providing payment mechanism was considered to be important in basic problems, opinion-based problems and competitive problems, since in all these problem types the platform should be able to deal with financial incentives, if any is provided by crowdsourcers. In problems that require expertise, it was considered to be insignificant, as experts deemed social incentives to be more motivating for experts than financial incentives. In collaborative fundraising problems it was considered to be trivial as there is usually no financial incentives involved, and experts unanimously stated that social incentives must be practiced in this crowdsourcing problem type.

Price negotiation was another feature that was always considered to be insignificant or trivial. This could indicate that crowdsourcers typically will not need to negotiate about financial incentives with their participants, as this procedure may be time-consuming and it may also demotivate those participants who do not find the results of negotiation as they initially expected. It was trivial in the case of collaborative fundraising problems, since there is obviously no financial incentives involved in this crowdsourcing problem type.

\section{E. Threats to Validity}

We conducted an expert survey involving a large number of experts, namely 37 , which is considered a reasonable number for such studies [31]. We added comment boxes to the survey questions so that experts can add more perception, which could also help us interpret the results. We also conducted the survey in an invitation-only manner which allowed only experts with a significant contribution in the field, demonstrated by research papers and projects applied in practice, to provide their opinions. Nonetheless, our study still has few threats to validity:

- The majority of experts came from academia which could imply that the results are academically biased. However, many experts had also applied crowdsourcing in practice, e.g., via case studies in mTurk or similar crowdsourcing platforms. This means that their opinions are not only based on theories, and are validated by some practical involvement.

- By analysing the current literature, we could identify five different problem types related to crowdsourcing. One might argue that there can be more problem types. Indeed, we do not claim completeness in this study. Furthermore, there may be new problem types in the future as well. On the other hand, the five problem types that were discussed in this paper are very common, if not the most common, problem types we could extract from the current literature.

- Given the complex nature of crowdsourcing and the heavy influence of human factors in it, we do not claim that these configurations are complete and/or never-changing. We acknowledge that some parts of the configuration for each crowdsourcing problem type may change with time and context. For example, financial incentives may become less or more important over time for certain crowdsourcing problem types, depending on the context where the problem type is being applied and the crowd which is recruited for that specific problem type. However, we also advocate that certain features are crucial to crowdsourcing problem types and are inherent to it. For example, largeness is inherently a feature of any opinion-based crowdsourcing problem type because it reduces the chances of bias and facilitates the broader view on the crowdsourcing problem type.

\section{CONCLUSION}

In this paper, we identified five common problem types in the domain of crowdsourcing and then conducted an expert survey to find the suitable crowdsourcing features for every problem type. Our study illustrates that different crowdsourcing problem types should employ different crowdsourcing 
features for optimal results. It also illustrates the need for a crowdsourcing configurator which can help crowdsourcers and developers in their choice of crowdsourcing features. Developing such a configurator will constitute the future work.

\section{ACKNOWLEDGMENT}

The research was supported by a European FP7 Marie Curie CIG grant (the SOCIAD Project) and Bournemouth University through the Graduate School Santander Grant for PGR Development.

\section{REFERENCES}

[1] J. Howe. (2006, June) Crowdsourcing: A definition. [Online]. Available: http://www.crowdsourcing.com/cs/2006/06/crowdsourcing_a.html

[2] M. Buhrmester, T. Kwang, and S. D. Gosling, "Amazon's mechanical turk a new source of inexpensive, yet high-quality, data?" Perspectives on Psychological Science, vol. 6, no. 1, pp. 3-5, 2011.

[3] D. C. Brabham, "Moving the crowd at threadless: Motivations for participation in a crowdsourcing application," Information, Communication \& Society, vol. 13, no. 8, pp. 1122-1145, 2010.

[4] P. Fraternali, A. Castelletti, R. Soncini-Sessa, C. Vaca Ruiz, and A. Rizzoli, "Putting humans in the loop: Social computing for water resources management," Environmental Modelling \& Software, vol. 37, pp. 68-77, 2012.

[5] P. Gay, C. Lehan, J. Moore, G. Bracey, and N. Gugliucci, "Cosmoquest: A cyber-infrastructure for crowdsourcing planetary surface mapping and more," in Proceedings of the 2014 Lunar and Planetary Institute Science Conference, vol. 45, 2014, p. 2927.

[6] L. Erickson, I. Petrick, and E. Trauth, "Hanging with the right crowd Matching crowdsourcing need to crowd characteristics." in Proceedings of the 2012 Americas Conference on Information Systems (AMCIS), 2012.

[7] K. Fort, G. Adda, and K. B. Cohen, "Amazon mechanical turk: Gold mine or coal mine?" Computational Linguistics, vol. 37, no. 2, pp. 413-420, 2011.

[8] A. Kittur, "Crowdsourcing, collaboration and creativity." ACM Crossroads, vol. 17, no. 2, pp. 22-26, 2010.

[9] Y. Singer and M. Mittal, "Pricing mechanisms for crowdsourcing markets," in Proceedings of the 22nd international conference on World Wide Web $(W W W)$. International World Wide Web Conferences Steering Committee, 2013, pp. 1157-1166.

[10] H. Simula, "The rise and fall of crowdsourcing?" in Proceedings of the 2013 46th Hawaii International Conference on System Sciences (HICSS), January 2013, pp. 2783-2791.

[11] D. E. Difallah, G. Demartini, and P. Cudré-Mauroux, "Mechanical cheat: Spamming schemes and adversarial techniques on crowdsourcing platforms." in Proceedings of CrowdSearch 2012 Workshop, 2012, pp. 26-30.

[12] C. Van Pelt and A. Sorokin, "Designing a scalable crowdsourcing platform," in Proceedings of the 2012 ACM SIGMOD International Conference on Management of Data, ser. SIGMOD '12. New York, NY, USA: ACM, 2012, pp. 765-766.

[13] M. Al Sohibani, N. Al Osaimi, R. Al Ehaidib, S. Al Muhanna, and A. Dahanayake, "Factors that influence the quality of crowdsourcing," in New Trends in Database and Information Systems II, ser. Advances in Intelligent Systems and Computing, N. Bassiliades, M. Ivanovic, M. Kon-Popovska, Y. Manolopoulos, T. Palpanas, G. Trajcevski, and A. Vakali, Eds. Springer International Publishing, 2015, vol. 312, pp. 287-300.
[14] G. M. Foody, "Rating crowdsourced annotations: evaluating contributions of variable quality and completeness," International Journal of Digital Earth, vol. 7, no. 8, pp. 650-670, 2014. [Online]. Available: http://dx.doi.org/10.1080/17538947.2013.839008

[15] S. M. Wolfson, "Crowdsourcing and the law," Master's thesis, Faculty of the Graduate School of the University of Texas, 2012.

[16] S. M. Wolfson and M. Lease, "Look before you leap: Legal pitfalls of crowdsourcing," in Proceedings of the 2011 American Society for Information Science and Technology, vol. 48, no. 1. Wiley Subscription Services, Inc., A Wiley Company, 2011, pp. 1-10.

[17] G. D. Saxton, O. Oh, and R. Kishore, "Rules of crowdsourcing: Models, issues, and systems of control," Information System. Management, vol. 30, no. 1, pp. 2-20, 2013. [Online]. Available: http://dx.doi.org/10.1080/10580530.2013.739883

[18] A. C. Rouse, "A preliminary taxonomy of crowdsourcing," in Proceedings of the 21st Australasian Conference on Information Systems (ACIS), 2010.

[19] D. Geiger, S. Seedorf, T. Schulze, R. C. Nickerson, and M. Schader "Managing the crowd: towards a taxonomy of crowdsourcing processes," in Proceedings of the 2011 Americas Conference on Information Systems (AMCIS), 2011.

[20] J. Pedersen, D. Kocsis, A. Tripathi, A. Tarrell, A. Weerakoon, N. Tahmasbi, J. Xiong, W. Deng, O. Oh, and G.-J. de Vreede, "Conceptual foundations of crowdsourcing: A review of is research," in Proceedings of the 2013 46th Hawaii International Conference on System Sciences (HICSS), January 2013, pp. 579-588.

[21] M. Hosseini, K. Phalp, J. Taylor, and R. Ali, "The four pillars of crowdsourcing: A reference model," in Proceedings of the 2014 IEEE Eighth International Conference on Research Challenges in Information Science (RCIS), May 2014, pp. 1-12.

[22] R. D. Fricker and M. Schonlau, "Advantages and disadvantages of internet research surveys: Evidence from the literature," Field Methods, vol. 14, no. 4, pp. 347-367, 2002.

[23] J. R. Evans and A. Mathur, "The value of online surveys," Internet Research, vol. 15, no. 2, pp. 195-219, 2005. [Online]. Available: http://www.emeraldinsight.com/doi/abs/10.1108/10662240510590360

[24] E. Estellés-Arolas and F. González-Ladrón-de Guevara, "Towards an integrated crowdsourcing definition," Journal of Information science, vol. 38, no. 2, pp. 189-200, 2012.

[25] D. G. Johnson, "Ethics online," Communications of the ACM, vol. 40, no. 1, pp. 60-65, January 1997

[26] D. Johnson, "Anonymity and the internet," The Futurist, vol. 34, no. 4, p. $12,2000$.

[27] N. Kaufmann, T. Schulze, and D. Veit, "More than fun and money. worker motivation in crowdsourcing-a study on mechanical turk," in Proceedings of the 2011 Americas Conference on Information Systems (AMCIS), 2011.

[28] D. Chandler and A. Kapelner, "Breaking monotony with meaning: Motivation in crowdsourcing markets," Journal of Economic Behavior \& Organization, vol. 90, no. 0, pp. 123 - 133, 2013.

[29] J. Rogstadius, V. Kostakos, A. Kittur, B. Smus, J. Laredo, and M. Vukovic, "An assessment of intrinsic and extrinsic motivation on task performance in crowdsourcing markets." in Proceedings of the 2011 International AAAI Conference on Web and Social Media (ICWSM), 2011.

[30] O. Tokarchuk, R. Cuel, and M. Zamarian, "Analyzing crowd labor and designing incentives for humans in the loop," IEEE Internet Computing, vol. 16, no. 5, pp. 45-51, September 2012.

[31] R. M. Cooke and K. N. Probst, Highlights of the expert judgment policy symposium and technical workshop. Resources for the Future Washington, DC, 2006. 